\title{
HISTORIA DE Diplodocus carnegii DEL MNCN: PRIMER ESQUELETO DE DINOSAURIO MONTADO EN LA PENÍNSULA IBÉRICA
}

\author{
Adán PÉREZ GARCÍA ${ }^{1,2}$ y Begoña SÁNCHEZ \\ CHILLÓN ${ }^{3}$
}

\begin{abstract}
${ }^{1}$ Departamento de Paleontología. Facultad de Ciencias Geológicas. Universidad Complutense de Madrid. C/ José Antonio Novais, 2. Ciudad Universitaria. 28040 Madrid, España. E. mail: paleontologo@gmail.com

${ }^{2}$ Unidad de Paleontología. Departamento de Biología. Universidad Autónoma de Madrid. C/ Darwin, 2. 28049 Cantoblanco, Madrid, España.

${ }^{3}$ Museo Nacional de Ciencias Naturales-CSIC. C/ José Gutiérrez Abascal, 2. 28006 Madrid, España. E. mail: mcnsc2b@mncn.csic.es
\end{abstract}

Pérez García, A. \& Sánchez Chillón, B. 2009. Historia de Diplodocus carnegii del MNCN: primer esqueleto de dinosaurio montado en la Península Ibérica. [History of Diplodocus carnegii of the MNCN: first assembled dinosaur skeleton in the Iberian Peninsula.] Revista Española de Paleontología, 24 (2), 133-148. ISSN 0213-6937.

\begin{abstract}
The arrival of a replica of the famous skeleton of Diplodocus donated by Andrew Carnegie to the Museo Nacional de Ciencias Naturales (Madrid) took place almost a century ago. It constitutes the first and unique skeleton of dinosaur assembled until the eighties of the past century in the Iberian Peninsula. This fact, the circumstances that surrounded their accomplishment, transports and installation, as well as the social and political repercussion that it had at the time, is detailed in the following pages. Besides using photographic documentation and unpublished handwritten documents, it has been collected the abundant references to the skeleton published in the Spanish press, which served as the link between paleontology and society.
\end{abstract}

Keywords: Paleontology, Diplodocus, Spanish press, political and social impact, Museo Nacional de Ciencias Naturales (MNCN).

\section{RESUMEN}

Hace casi un siglo tuvo lugar la llegada a Madrid de una de las famosas réplicas del esqueleto de Diplodocus, cedida por el Andrew Carnegie, al Museo Nacional de Ciencias Naturales. Constituye el primer y único esqueleto de un dinosaurio montado hasta la década de 1980 en la Península Ibérica siendo, durante todo ese tiempo, uno de los más importantes referentes que contribuyó a acercar la Paleontología a la población. Este hecho, las circunstancias que rodearon su realización, transporte e instalación, así como la repercusión social y política que generó, se detallan en las siguientes páginas. Para ello, además de emplear documentación manuscrita y fotográfica inédita, se recopilan las abundantes alusiones a este esqueleto publicadas en la prensa española, que sirvieron de nexo de unión entre la Paleontología y la sociedad.

Palabras clave: Paleontología, Diplodocus, prensa española, repercusión política y social, Museo Nacional de Ciencias Naturales (MNCN).

\section{INTRODUCCIÓN Y OBJETIVOS}

El esqueleto de Diplodocus carnegii Hatcher, 1901 de la Colección de Paleontología de Vertebrados del Museo Nacional de Ciencias Naturales (Madrid), cuyo número de catálogo es MNCN 63731, es una réplica del esqueleto conocido como "Dippy" perteneciente al Carnegie Museum of Natural History (Pittsburgh). En julio de 1899 se informaba al Museo de Pittsburgh, mediante un telegrama, del descubrimiento realizado por Arthur Coggeshall, el día 4 de ese mes, de un un esqueleto bastante completo de un gran saurio jurásico en la Formación Morrison, concretamente en Sheep Creek (Quarry D), en Wyoming. Durante ese año A. Coggeshall, J. L. Wortman, W. H. Reed y W. 
C. Reed excavaron este esqueleto, que sería el holotipo de la especie Diplodocus carnegii. Al año siguiente, en el mismo yacimiento, Peterson y Gilmore extrajeron otro ejemplar más incompleto perteneciente a un individuo de tamaño similar, que constituyó el paratipo de este taxón. Todo este material se llevó al Carnegie Museum, donde se preparó para realizar un montaje de un esqueleto de Diplodocus. En el montaje se utilizaron huesos de varios individuos, ya que ninguno de ellos estaba completo (Cabrera, 1913a; Mc Intosh, 1981). El esqueleto más completo era el descubierto por Coggeshall, siglado como CM 84, compuesto por vértebras cervicales, dorsales, sacras, costillas, escápula y coracoides izquierdos, ilion derecho y parte del izquierdo, pubis, isquion, fémur derecho, placas esternales y clavícula. A éste se añadieron vértebras caudales medias y escápula, coracoides, tibia, fíbula y pie derechos del ejemplar encontrado al año siguiente (CM 94) y las vértebras caudales distales del ejemplar CM 307, Diplodocus sp., que había sido extraído de otro yacimiento en la Formación Morrison (Quarry B) por Utterback en 1903. La pata delantera derecha y, en el caso de las copias realizadas también la izquierda, fueron modeladas a partir del individuo de menor tamaño CM 662, más tarde asignado a Diplodocus hayi Holland, 1924, constituyendo el ejemplar tipo de esta especie, que había sido recolectado por Utterback en Quarry A entre 1902 y 1903. De este ejemplar sólo se conocía parte del cráneo, que se combinó con el fragmento correspondiente del ejemplar USNM 2673 para poder modelar el cráneo de "Dippy". Algunos de los metápodos y falanges se modelaron a partir del ejemplar de Camarasaurus del AMNH 965 (Mc Intosh, 1981).

Una copia de este esqueleto constituiría el ejemplar que fue instalado en Madrid en 1913. El análisis de la documentación inédita (Anexo 1), de fotografías de la época y de las noticias que fueron publicadas en la prensa española, permite conocer todos los detalles acerca de cómo fueron posibles su llegada e instalación. El montaje de este esqueleto, así como su solicitud, realización y envío, tuvieron una importante repercusión social, acercando por primera vez la Paleontología a la población española.

Abreviaturas: ACN, Archivo del Museo Nacional de Ciencias Naturales (Madrid). AMNH, American Museum of Natural History (New York). BN, Biblioteca Nacional (Madrid). CM, Carnegie Museum of Natural History (Pittsburgh). CN, Dinosaur National Monument (Colorado, Utah). MNCN, Museo Nacional de Ciencias Naturales (Madrid). USNM, National Museum of Natural History (Smithsonian Institution, Washington).

\section{ANTECEDENTES}

El director del Museo de Pittsburgh, William Jacob Holland, que fue el primero en analizar algunos de los hue- sos del ejemplar CM 84 (Holland, 1900), envió un croquis al multimillonario Andrew Carnegie de cómo podría quedar montado el esqueleto de Diplodocus. El magnate, que había subvencionado las excavaciones y la Institución, se encontraba veraneando en su castillo de Escocia cuando el rey Eduardo VII de Inglaterra vio el dibujo de Diplodocus colgado en una de las salas de su residencia, indicando su deseo de tener un esqueleto igual en su país. Dado que encontrar otro Diplodocus era una labor casi imposible, Carnegie pensó que se podría hacer una reproducción del esqueleto de "Dippy" con la que homenajear al monarca (Cabrera, 1913a). La réplica fue instalada en el British Museum of Natural History de Londres en mayo de 1905. Ante este hecho, otros países americanos y europeos solicitaron copias del mismo a lo largo del siguiente decenio. Así pues, fueron regaladas a los jefes de Estado de Inglaterra, Alemania, Francia, Austria, Italia, Rusia, Argentina, España y México, pasando a formar parte de los Museos de Historia Natural de Londres, Berlín, París, Viena, Bolonia, San Petersburgo, La Plata, Madrid y Ciudad de México respectivamente. La realización, envío e instalación de los esqueletos no fue sencilla, requiriendo un tiempo relativamente largo, además de elevadas cantidades de dinero. De hecho, en el caso del ejemplar regalado a España, pasaron algo más de dos años desde su solicitud hasta su exposición al público, que tuvo lugar a finales de 1913. En esa época apenas se tenía registro de dinosaurios ni de otros reptiles mesozoicos en ninguna institución española. Los pocos fósiles reconocidos eran un supuesto diente de Megalosaurus del Jurásico de Asturias, identificado como tal por Egozcue (1873), algunos huesos aislados de dinosaurios y cocodrilos que, procedentes del Cretácico Inferior de las localidades valencianas de Buñol y Morella, formaban parte de la colección de la Facultad de Ciencias de la Universidad Literaria de Valencia (Pérez García et al., 2009) y, finalmente, los ejemplares que formaban parte de las colecciones del MNCN. Estos últimos consistían en huesos de ictiosaurios, plesiosaurios, cocodrilos, tortugas y otros reptiles indeterminados, que habían sido obtenidos en algunos países europeos por Juan Vilanova y Piera y Augusto González de Linares (Sánchez Chillón \& Pérez García, 2008), el recién descubierto Lariosaurus balsami del Triásico de Estada, Huesca (Ferrando, 1912), los fragmentos de huesos del Cretácico Inferior de Morella (Castellón) y de Utrillas (Teruel) que habían sido atribuidos por Vilanova al ornitópodo Iguanodon (Vilanova y Piera, 1872,1873) y un caparazón de tortuga del Cretácico Inferior de Morella.

El de Diplodocus fue el primer y único esqueleto de dinosaurio montado en la Península Ibérica durante más de 70 años, hasta el montaje del Triceratops de L'Institut de Paleontologia Miquel Crusafont de Sabadell en el año 1982. 


\section{SOLICITUD DE RÉPLICAS DE GRANDES ESQUELETOS DE DINOSAURIOS PARA EL MNCN}

Tanto las noticias del descubrimiento de los ejemplares de Diplodocus como el montaje del esqueleto, o el regalo de copias del mismo a distintos jefes de Estado europeos, son citadas en múltiples ocasiones en la prensa española (Ver por ejemplo: Anónimo, 1899, 1900a; Cabrera, 1913b). También son muchos los científicos que tienen relación con personal del MNCN y que informan de estos hechos. Por ejemplo, el 8 de noviembre de 1911 se comunica a la Real Sociedad Española de Historia Natural, la rápida visita de miembros de esta Sociedad, en el mes de julio, al Muséum National d'Histoire Naturelle de París, en el que tienen la ocasión de contemplar el esqueleto de Diplodocus carnegii cuando regresan de la segunda Asamblea General de la Asociación Internacional de Sismología, celebrada en Manchester (Sánchez-Navarro Neumann, 1911). Con motivo de esta asamblea visitan también el British Museum of Natural History de Londres, donde observan la primera réplica de Diplodocus. Manuel María Sánchez-Navarro Neumann, director del Observatorio de La Cartuja, tomó apuntes en ambos museos, en especial de la sección de Paleontología (Espinar Moreno, 2002).

Al ser un hecho tan conocido el regalo de réplicas de Diplodocus a los jefes de Estado de distintos países, Ignacio Bolívar y Urrutia, director del MNCN, escribe al Ministro de Estado, Manuel García Prieto, Marqués de Alhucemas, el 28 de octubre de 1911. Mediante esa carta pretende conseguir una petición oficial, firmada por el Monarca, Alfonso XIII, de dos réplicas de grandes esqueletos de dinosaurios: un Iguanodon de los que se encontraban montados en el Museo de Bruselas y un Diplodocus, cuyo esqueleto original estaba montado en el Museo de Pittsburgh. La primera petición ya se había realizado previamente desde el MNCN el 14 de marzo de 1904, cuando Bolívar realizó las gestiones necesarias para conseguir que el Museo del Congo, Museo Tervueren, situado en Bélgica, le cediera los mamíferos del Congo que tuviera duplicados, especialmente un okapi, y para que el Museo Real de Historia Natural de Bruselas hiciera lo mismo con un molde de Iguanodon "de los que este Museo facilita y que están vaciados sobre los ejemplares típicos que constituyen la más envidiable riqueza del referido Museo". La solicitud del okapi había sido concedida pero no la de Iguanodon, justificándose por su excesivo coste, "mil quinientos francos sin contar la armazón de hierro necesaria para sostener la inmensa mole de ese esqueleto" que el Museo de Bruselas no era en esos momentos capaz de asumir, aunque sí lo había hecho en otras ocasiones. Para adquirir este molde o el de cualquier otro ejemplar científico, se indicó que se debería abonar su precio o, preferentemente, intercambiarlo por otros objetos. No obstante, el director del Museo de Bruselas, Edouard Dupont, propuso dejar las negociaciones para más adelante, debido al traslado a un nuevo edificio al que se estaban sometiendo entonces todas las colecciones (ACN0273/017).

Según Bolívar, el director del Museo de Pittsburgh, W. J. Holland, manifestó que Mr. Andrew Carnegie, "el célebre potentado yanqui a quien pertenece el Museo", no se negaría a conceder el molde de Diplodocus si la petición se realizara con carácter oficial, como regalo a sus monarcas, tal como había ocurrido en otros países como con el caso del Rey Eduardo VII de Inglaterra y el Kaiser Guillermo II de Alemania. Manuel García Prieto, Ministro de Estado, responde a Bolívar el 6 de noviembre de 1911 informándole que había escrito a los representantes del Rey en Bruselas y Washington para que averiguaran las condiciones en que los Museos de Bruselas y Pittsburgh facilitarían los moldes de los dos reptiles. También le pregunta si el MNCN podría correr con los posibles gastos (ACN0303/001, doc.1, doc.2; ACN0303/002/001, doc.1, doc.2). El 14 de noviembre Bolívar agradece la rápida respuesta y el interés demostrado por García Prieto, añadiendo que el MNCN se hará cargo de los gastos del transporte de los ejemplares (ACN0303/001, doc.3; ACN0303/002/001, doc.3). El 30 de noviembre el Ministro de España en Bruselas escribe al Ministro de Estado en Madrid, carta que llega a Bolívar el 4 de diciembre. En la respuesta, el Museo de Historia Natural de Bruselas indica que no es posible satisfacer la petición en ese momento, ya que necesitan la colaboración de personal extranjero para realizar la copia de Iguanodon. Teniendo en cuenta que comporta un gran riesgo de las matrices de yeso, y unido al coste y esfuerzo para su realización, este encargo no podría realizarse hasta haber recibido al menos seis peticiones. En la carta se indicaba, además, que existían otras prioridades puesto que, tras el traslado del Museo a los nuevos locales, aún no se habían podido colocar las colecciones. Con la petición del MNCN eran ya cuatro las que estaban en lista de espera, pero al menos antes de un año no se podría responder a estas solicitudes. El Director del Museo de Historia Natural de Bruselas, Gustave Gilson, promete que el primer molde que se realice será para España (ACN0303/001, doc.4, doc.5). Unos años después, cuando se pretendía cumplir con este encargo, la Primera Guerra Mundial impidió su ejecución "interrumpiendo la labor pacífica y provechosa de la Ciencia" (Bolívar y Urrutia, 1915). En 1925, cuando el paleontólogo José Royo y Gómez visitó el Museo de Historia Natural de Bruselas, volvió a tratar con Louis Dollo la solicitud del vaciado de un esqueleto de Iguanodon. Aunque obtuvo la promesa de que dicho molde sería facilitado (Royo y Gómez, 1925) esto nunca llegó a ocurrir.

El 8 de enero de 1912, Manuel García Prieto vuelve a escribir a Bolívar confirmándole que se disponía ya de la autorización del Rey, por lo que había ordenado a Juan Riaño, Ministro de "Su Majestad" en Washington, solicitar 
a Carnegie el molde de Diplodocus, según Real Orden $\mathrm{n}^{\circ} 8$ de fecha 4 de enero de 1912 (ACN0303/002/001, doc.11). El 27 de enero Riaño recibe la respuesta de Carnegie, en la que se informa que ya se ha ordenado la reproducción del esqueleto de Diplodocus, estimando que tardará seis o más meses en estar terminada, haciendo a su vez partícipe de esta noticia a Manuel García Prieto (ACN0303/002/001, doc.7). El 14 de febrero de 1912, el Ministro de Estado comunica esta noticia a Bolívar, que se había interesado a través de una carta escrita el 20 de enero, adjuntándole además la correspondencia que le había sido enviada por Riaño y Holland (ACN0303/002/001, doc.4). Mes y medio más tarde, el 29 de abril de 1912, Holland se pone en contacto con Bolívar, confirmándole que se estaba realizando una reproducción de Diplodocus carnegii idéntica a las regaladas al Museo Británico y a otros museos de $\mathrm{Eu}-$ ropa, labor que no se terminaría hasta finales de año, por lo que calculaba que al menos pasarían doce meses antes de que fuera remitida a Madrid y colocada. En esta carta le indica además las medidas del dinosaurio, con intención de saber si se dispone de una sala en Madrid lo suficientemente amplia o si, por el contrario, se ha de curvar la cola, dato que necesita tener en cuenta para la "confección de los hierros y soportes de la cola". Pone como ejemplos los Diplodocus con la cola extendida montados en Inglaterra, Italia y Alemania o los de París y San Petersburgo, en los que la falta de espacio de la sala había obligado a montar la cola con una cierta curvatura. Por ese motivo, solicita un plano de la sala donde será ubicado el ejemplar. Confirma que el gasto de la fabricación de las plataformas sobre las que irá colocado, al igual que su instalación, correría a cargo de Andrew Carnegie, quien también se encargaría de los posibles gastos derivados de su transporte. El personal del MNCN ha de responsabilizarse de la fabricación de esas peanas, según indicaciones que se remitirán más adelante, para que estén listas cuando llegue el ejemplar y facilitar así su rápido montaje (ACN0303/002/001, doc.9). En su contestación, Bolívar le asegura que procurará disponer de una sala del tamaño necesario (ACN0303/002/001, doc.13).

\section{EL TRANSPORTE Y EL MONTAJE DEL ESQUELETO DE DIPLODOCUS}

El 12 de junio de 1913 Holland vuelve a dirigirse por carta a Ignacio Bolívar, informándole de que casi está terminada la reproducción, que en breve se comenzará a embalar. Holland realizará los trámites necesarios a fin de obtener el transporte marítimo hasta España gratis, aconsejando a Bolívar que realice las negociaciones equivalentes para que también el traslado en tren hasta Madrid sea gratuito, como había ocurrido en el caso del envío al resto de países que habían recibido anteriormente semejante donación, con motivo de tratarse de un regalo especial destinado a la Casa Real. Indica que alguien deberá estar preparado a su llegada a puerto, para ocuparse de los trámites de su desembarco y su traslado a Madrid. Holland irá a Madrid para realizar la instalación con uno de sus ayudantes, Arthur Coggeshall, descubridor del primero de los esqueletos que forman parte del ejemplar (CM 84), en ese momento jefe del Laboratorio de Paleontología del Carnegie Museum. Propone los meses de septiembre y octubre como fechas más probables para efectuar el viaje (ACN0275/017, doc.2; ACN0303/002/001, doc.13).

El Museo Nacional de Ciencias Naturales, ubicado en su sede actual de la calle José Gutiérrez Abascal desde 1907, disponía por aquellas fechas de menos espacio en el edificio que en la actualidad, ya que además de compartirlo con la Escuela de Ingenieros Industriales, que entonces ocupaba dependencias parecidas a las actuales en lo que se refiere al edificio principal, el MNCN compartía además el edificio con el Museo del Traje, situado en lo que en la actualidad constituyen las Salas de Paleontología y Geología en el ala sur del edificio, y con la Guardia Civil, que a su vez ocupaba una parte de este ala sur. Viendo que la llegada del saurio era inminente y que aún no se contaba con una sala donde montarlo, Ignacio Bolívar, a través del Ministro de Instrucción Pública, realiza una petición al Rey Alfonso XIII el 7 de julio de 1913, reiterándose la solicitud el día 28 , de un local suficientemente amplio para exponer el ejemplar, sugiriendo que una buena opción podía ser "el gran salón o rotonda del Palacio de la Industria y de las Artes", propiedad de la Escuela de Ingenieros Industriales, situado en el ala norte del edificio y rodeado por las dependencias del MNCN. Eduardo Hernández-Pacheco, secretario del Museo, junto con Ignacio Bolívar solicitan audiencia a Alfonso XIII "con el deseo de expresar a Su Majestad nuestra gratitud por la protección que se digna dispensar a este centro y enterarle de la llegada del regalo de Mr. Carnegie" (ACN0275/017, doc.1; ACN0303/002/001, doc.15). Al día siguiente, el Presidente del Consejo de Ministros promete a Bolívar hablar con el Ministro de Instrucción Pública para llegar a un acuerdo sobre el local requerido con los Ingenieros Industriales, a quien pertenece la sala. El mayor problema para su adjudicación radica en la ausencia de una partida presupuestaria destinada a este fin (ACN0303/002/001, doc.16).

El 8 de agosto de 1913 Holland vuelve remitir a Bolívar los planos de las peanas ya que, al parecer y tal como indicaba Bolívar por carta el 25 de julio, éstos no habían llegado a Madrid. En la misma, Holland realiza una serie de advertencias sobre como montar dichas peanas. Le envía dos planos: el empleado en el montaje de Viena, donde se tuvo que curvar la cola, y el enviado para el montaje en Bolonia, donde había espacio para instalarlo con la cola extendida. De esta manera se podían ver las relaciones de anchura y longitud que debían tener las peanas en cada uno de los casos. El ejemplar estaba ya embalado y listo para enviarse, quedando encargado Holland 
de solucionar los trámites de transporte a España desde Nueva York e informar, cuando lo supiera, del puerto de destino (ACN0303/002/001, doc.17). La construcción e instalación de las peanas, encargadas en una carpintería madrileña, Arroyo, Gallego y Cía., supone un gasto de 1.625 pesetas (ACN0275/017, doc.13; ACN0303/002/001, doc.59). El 12 de agosto de 1913, Alfonso XIII concede finalmente el dinero necesario para la ampliación del local, que se realizaría a través del Ministerio de Hacienda (ACN0303/002/001, doc.19).

La réplica del esqueleto sale por fin desde el puerto de Nueva York el 5 de septiembre de 1913. El envío lo constituyen 34 cajas con un peso total de 4.082 kilogramos, siendo trasladado en el vapor Montserrat de la Compañía Trasatlántica Española (Fig. 1) y llegando al puerto de Barcelona el 19 de septiembre (ACN0275/017, doc.5; ACN0303/002/001, doc.20). Tal como se había prometido, Carnegie abona todos los gastos del transporte hasta Nueva York y del embarque (ACN0275/017, doc.3). El Marqués de Comillas, Presidente de la Compañía Trasatlántica Española, concede el transporte gratuito "en atención a las especiales circunstancias que en él concurren” (ACN0275/017, doc.5; ACN0303/002/001, doc.26). Bolívar realiza las gestiones necesarias para que se manifieste oficialmente, en nombre de la Junta de Profesores del Museo, el agradecimiento al Marqués de Comillas por la condonación de los gastos del flete, cosa que éste agradece (ACN0275/017, doc.7, doc.8, doc.9). La persona encargada de resolver los trámites desde el puerto de Barcelona es el Profesor del Instituto General Técnico de Barcelona,

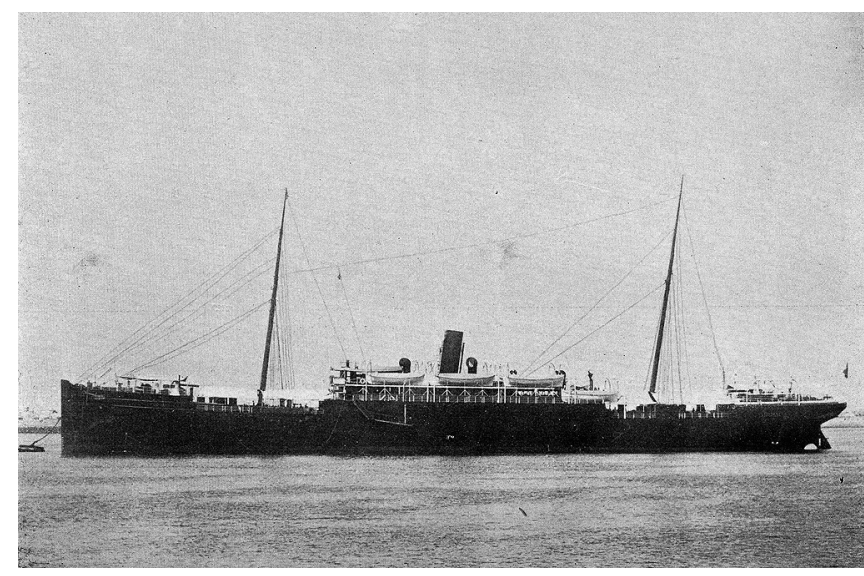

Figura 1. El vapor Montserrat, de la Compañía Trasatlántica Española. En este barco viajó el esqueleto de $D i$ plodocus, en septiembre de 1913, de Nueva York a Barcelona. Imagen publicada en la revista El Mundo Naval Ilustrado en 1900 (Anónimo, 1900b). The vapour Montserrat, of the Compañía Trasatlántica Española. On this ship the skeleton of Diplodocus travelled from New York to Barcelona, in September 1913. Image published in the magazine El Mundo Naval Ilustrado in 1900 (Anónimo, 1900b).
Manuel Cazurro. Es él a quien Bolívar encomienda conseguir que los costes de aduana sean gratuitos por tratarse de material científico destinado a un establecimiento oficial (ACN0303/002/021). Durante septiembre y octubre, Cazurro se encarga de este objetivo, consiguiéndose que se reduzcan los gastos. Para ello, lo primero que hace es ordenar el traslado de las cajas del muelle a la aduana, por estar allí más seguras y por ahorrarse así 14 pesetas al día por su almacenaje y vigilancia. También consigue que sean gratuitos los derechos de arancel, que hubiesen supuesto más de 800 pesetas, por constituir su tarifa a razón de 20 pesetas por cada 100 kilogramos (ACN0303/002/001, doc.23, doc.24, doc.25). El 21 de septiembre Bolívar solicita al Subsecretario de Instrucción Pública y Bellas Artes que realice las gestiones pertinentes para conseguir que el transporte ferroviario hasta Madrid sea también gratuito, escribiendo él mismo al director asociado de la Compañía de Ferrocarriles (ACN0275/017 doc.3, doc.10). La Compañía de Ferrocarriles manifiesta que no puede conceder el trasporte gratuito pero que aplicará la menor tarifa para el traslado del ejemplar al MNCN a la mayor velocidad posible, y le informa que, sin embargo, hasta no obtener las franquicias de aduanas y del flete el ejemplar no podrá ser enviado a Madrid (ACN0303/002/001, doc.33, doc.36, doc.37, ACN0303/002/001, doc.23, doc.24, doc.25).

Las noticias del regalo del esqueleto de Diplodocus se difunden por la prensa española, publicándose información de su llegada al puerto de Barcelona, su inminente traslado a Madrid y la problemática del local donde sería instalado por el propio director y operarios del Museo de Pittsburgh. En estos artículos se destaca también la generosidad de Carnegie así como de la Compañía Trasatlántica Española (ACN0303/002/022; ACN0303/002/054; Anónimo, 1913g, 1914a; Lorenzo, 1913). La noticia es aprovechada igualmente para promocionar algunos productos, como los Bombones y Caramelos ;Lagarto, lagarto!:

“Acabo de leer, y aún estoy loco,
que al Museo de Ciencias Naturales
van a traer los huesos colosales
de un lagarto llamado «Dioplodoco»
Tres toneladas pesa, que no es poco;
Y treinta metros mide, no cabales;
Procede de los tiempos diluviales;
Y asusta, dicen, como asusta el “coco (...)”
(ACN0303/002/001, doc.53).

La difusión de esta noticia aumenta y su repercusión social se deja notar en la prensa, aprovechándose incluso como medio para realizar públicamente críticas a la clase política, de las que no se libra ni el mismo Ministro de Instrucción Pública (Anónimo, 1913d, 1913e; Lorenzo, 1913).

El Director de la Escuela Central de Ingenieros Industriales pide a Ignacio Bolívar, el 6 de noviembre de 1913, que la sala donde se pretende instalar el Diplodocus quede 


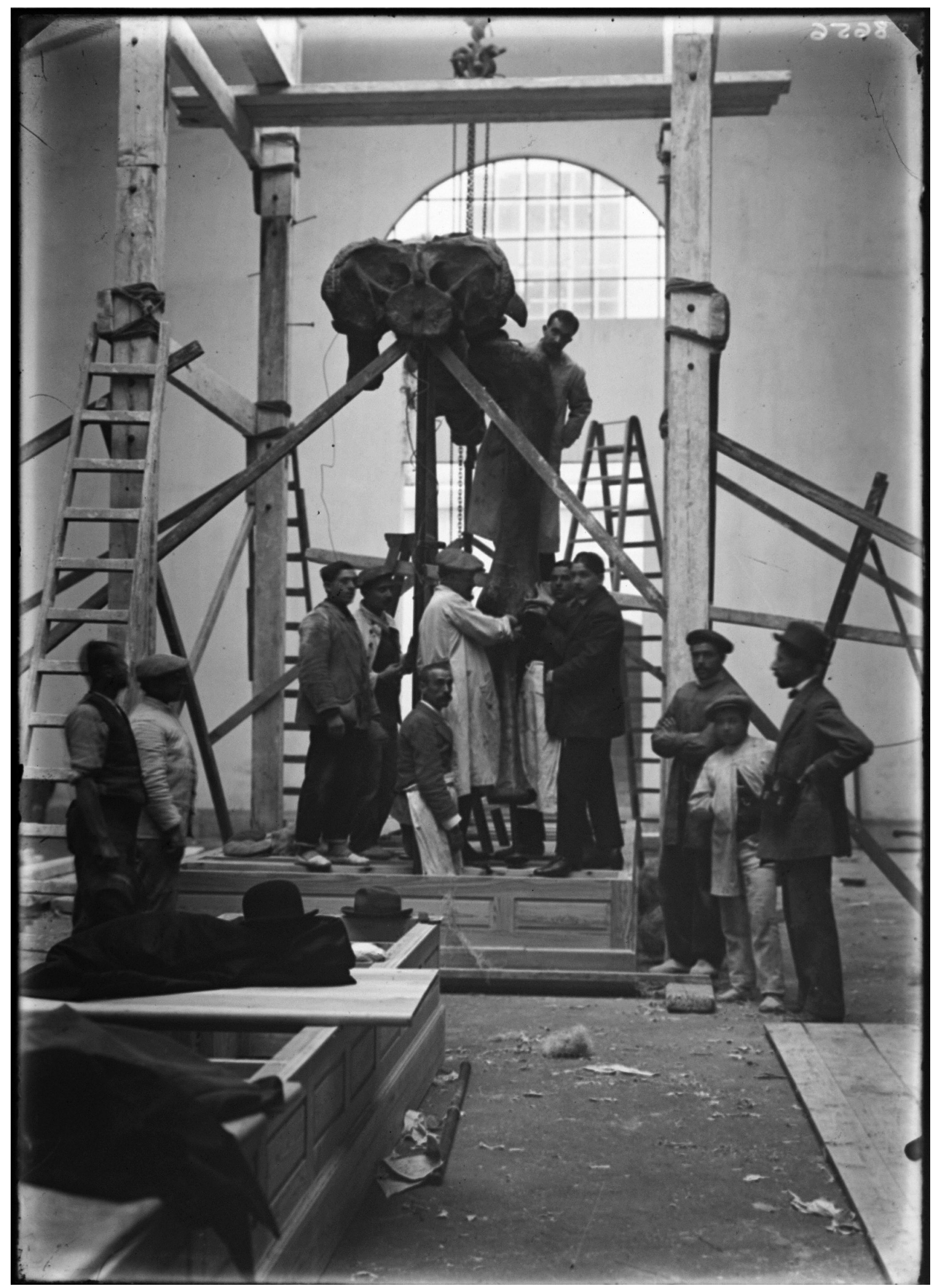


incomunicada de la Escuela de Ingenieros Industriales y unida al resto de locales que constituían el MNCN puesto que, hasta ese momento, el acceso a esta sala se debía realizar necesariamente por dicha Escuela. Hasta que se pueda efectuar la obra para unir ambos locales, el horario de visitas de esta sala estará muy limitado, autorizándose el paso por la Escuela de Ingenieros Industriales únicamente en días no lectivos, es decir, los domingos y días festivos, de 10 a 13 horas (ACN0303/002/001, doc.35). El 10 de noviembre esta nueva sala, que hasta el momento había sido el Pabellón de Cristal de la Escuela de Ingenieros, es visitada por Francisco Bergamín García, Ministro de Instrucción Pública y Bellas Artes, a quien se había solicitado previamente su colaboración para obtener dicho local. En esa visita se comprueba que la sala es adecuada y que en ella deben realizarse únicamente algunas obras para adaptarla a su nuevo fin, obras que serían llevadas a cabo bajo la dirección del arquitecto Enrique Repullés y Segarra (ACN0303/002/001, doc.52; ACN0275/017, doc.12, doc.14).

El 11 de noviembre William Holland y Arthur Coggeshall llegan a Madrid, alojándose en el Hotel Ritz (ACN0303/002/001, doc.49). Ese mismo día, Manuel Cazurro, cuya función era controlar y conseguir el traslado de las 34 cajas desde la Aduana de Barcelona, informa a Bolívar de que el esqueleto de Diplodocus ha salido ya con destino a Madrid al haberse obtenido la franquicia del flete y estar arregladas las vías férreas, que habían sufrido daños por los temporales: "Felizmente creo que a estas horas el Diplodocus llevado por otro monstruo mayor y más ligero que él estará rodando camino de Madrid" (ACN0303/002/001, doc.27, doc.50). Los gastos generados desde su llegada al puerto de Barcelona hasta el transporte al Museo corresponden a 761,25 pesetas, siendo 193,10 pesetas de gastos de aduana, 478,90 de gastos del transporte en tren de Barcelona a Madrid, 69 de traslado en camión desde la estación de tren de Atocha al Museo, 20 de gastos por 4 hombres para realizar este transporte y 0,25 pesetas de sellos (ACN0275/017, doc.13; ACN0303/002/001, doc.51). Todos estos gastos son también pagados por Carnegie. Desde el día 12 de noviembre y hasta el 28 de ese mismo mes, tiene lugar el montaje del esqueleto por parte de Holland y Coggeshall, ayudándose por medio de la construcción de una estructura de andamios de madera, actividad en la que prestan su colaboración parte del personal del MNCN, entre los que destaca Ángel Cabrera (Fig. 2). También la prensa española se hace eco de esta instalación, publicándose una fotografía de este proceso en La Ilustración Española y Americana el día 8 de diciembre, en la que se da cuenta de la noticia sobre su montaje "De este modo, la enorme osamenta del reptil, una osamenta de veinticuatro metros de longitud, quedó montada en una de las salas de aquel soberbio establecimiento científico, siendo el asombro de los hombres de ciencia y la envidia de los demás museos del mundo" (Cabrera, 1913a) (Fig. 3).

\section{PRESENTACIÓN OFICIAL DE DIPLODOCUS}

Concluida la instalación del esqueleto de Diplodocus el 28 de noviembre de 1913, se llevaron a cabo una serie de actos oficiales, entre los cuales se encuentra la celebración de una Sesión Extraordinaria de la Real Sociedad Española de Historia Natural, con el propósito de nombrar a Holland socio honorario de la misma, cubriendo asî la plaza que había quedado vacante por el fallecimiento del naturalista británico Lord Abevury Mr. John Lubbock. Igualmente tuvo lugar el nombramiento de Coggeshall como socio correspondiente extranjero (Díaz del Villar, 1913). La inclusión en la lista de estos dos nuevos socios tuvo lugar en la publicación del número 14 del Boletín de la Real Sociedad Española de Historia Natural, el 10 de Enero de 1914.

Esa misma noche Holland impartió una conferencia en el Instituto Internacional de Madrid. En ella y mediante numerosas proyecciones, Holland mostró al público asistente los distintos departamentos del Museo de Pittsburgh y las labores de recolección, preparación e investigación de fósiles allí realizadas. Se centró en los reptiles jurásicos, especialmente en el propio Diplodocus, mostrando imágenes de su hallazgo, extracción, reconstrucción del esqueleto y de cómo sería este animal en vida (Fig. 4). Las explicaciones se realizaron en inglés, siendo traducidas al castellano por Ángel Cabrera, colector del MNCN y miembro de la Sociedad Española de Historia Natural. A este acto asistieron casi todos los socios de dicha Sociedad, así como un público selecto, que respondieron con numerosos aplausos a la disertación de Holland (Anónimo, 1913c).

El mismo 28 de noviembre, Ignacio Bolívar recibe una carta de El Marqués de Aguilar, Secretario Particular de la

Figura 2. Montaje del esqueleto del Diplodocus carnegii en el MNCN, en vista posterior, con parte de la cintura pelviana, el fémur y la tibia derechos. Subidos en la peana se distinguen: el segundo por la izquierda a un jovencísimo José Royo y Gómez, en el centro, con la bata, Eduardo Hernández-Pacheco y a la derecha Luis Lozano. Bajo la peana, el primero por la izquierda es Arthur Coggeshall. Fotografía tomada entre el 12 y el 28 de noviembre de 1913 (Foto: ACN 8656). Assembly of the skeleton of Diplodocus carnegii in the MNCN. Posterior view, showing part of the pelvis and the right femur and tibia. Up to the pedestal one can distinguish, the second on the left a young José Royo y Gómez, in the centre with the white coat Eduardo Hernández-Pacheco, and on the right Luis Lozano. Down, on the floor, the first on the left is Arthur Coggeshall. The photograph was taken between 12 and $28^{\text {th }}$ November of 1913 (Photo: ACN 8656). 


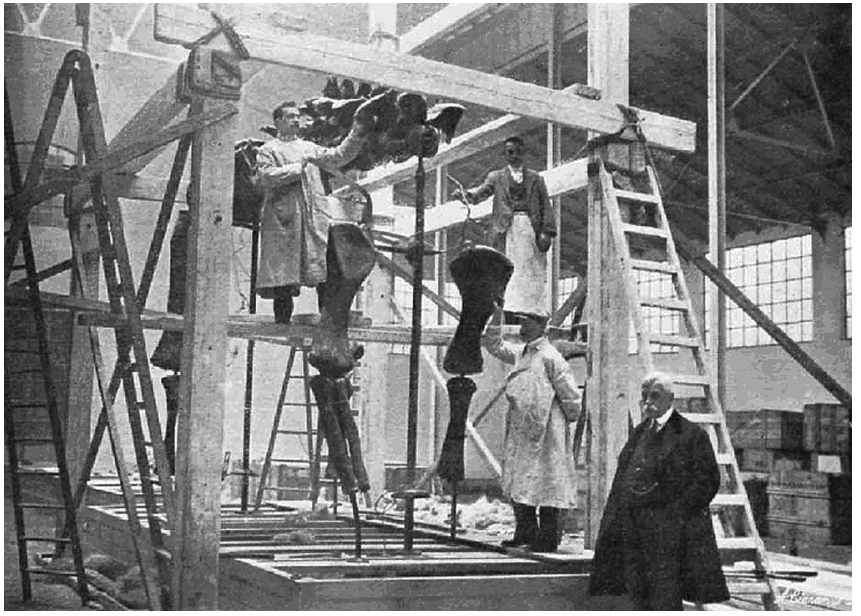

Figura 3. Fotografía del montaje del Diplodocus en el MNCN visto desde la parte anterior del animal. Esta imagen fue publicada en La Ilustración Española y Americana el 8 de diciembre de 1913 (Rivero, 1913). El ella se puede distinguir a William J. Holland, en primer plano, y a Ángel Cabrera, sobre un andamio, en la parte izquierda de la fotografía. Al fondo se observan algunas de las 34 cajas de madera en las que viajó el esqueleto. Por cortesía de la Hemeroteca Nacional (BNE).

Photograph of the assembly of the Diplodocus in the $M N C N$ seen from the front part of the animal. This image was published in La Ilustración Española y Americana on December $8^{\text {th }}, 1913$ (Rivero, 1913). It can be distinguishing William J. Holland, in the foreground, and Ángel Cabrera, on scaffolding on the left side of the photograph. In the background there are some of the 34 wooden boxes in which the skeleton travelled. Courtesy of the Hemeroteca Nacional of Spain (BNE).

Reina María Cristina, en la que le agradece en nombre de la Reina todas las informaciones referentes al transcurso del montaje del esqueleto así como las gestiones realizadas (ACN0303/002/058, doc.58).

El 29 de noviembre se expone el esqueleto de Diplodocus al público (Anónimo, 1913c), acontecimiento que es comunicado por Bolívar al Ministro de Instrucción Pública y Bellas Artes el 5 de diciembre (ACN0275/017, doc.13).

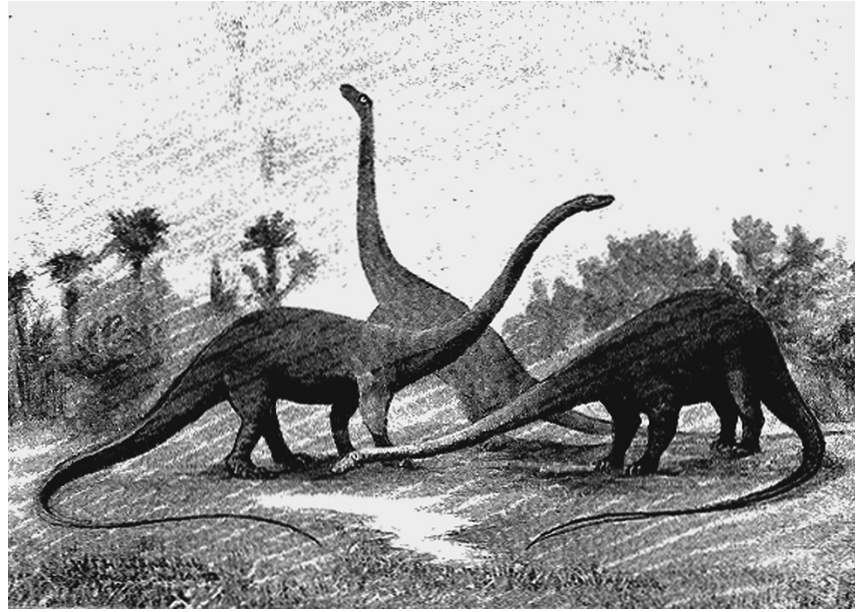

Figura 4. Interpretación del aspecto que tendría Diplodocus en vida, realizada por William J. Holland. Este dibujo se empleó para ilustrar una de las noticias publicadas en la prensa española sobre el regalo de Carnegie. Tomada de la revista Blanco y Negro, 7 diciembre de 1913 (Anónimo, 1913f). Por cortesía de la Hemeroteca Nacional (BNE).

Interpretation of Diplodocus would look at life, made by William J. Holland. This drawing was used to illustrate one of the news published in the Spanish press about the Carnegie gift. Taken from Blanco y Negro, December $7^{\text {th }}, 1913$ (Anónimo, 1913f). Courtesy of the Hemeroteca Nacional of Spain (BNE).

Dos días más tarde vuelve a escribirle con motivo de la solicitud de dinero para poder instalar vitrinas y armarios donde alojar las colecciones de Paleontología en la nueva sala (ACN0275/017, doc.14). Pero quizá el acontecimiento social más relevante es su inauguración por parte de la Casa Real el día 2 de diciembre. En su visita a la nueva sala asisten la Reina María Cristina y la Infanta Beatriz, que contemplan el esqueleto de Diplodocus. Por parte del MNCN, son Ángel Cabrera, Francisco Ferrer e Ignacio Bolívar quienes hacen de anfitriones de esta visita, cuya repercusión tuvo eco en la prensa, publicándose una fotografía en $A B C$ el 3 de diciembre (Anónimo, 1913b, 1913f) (Fig. 5).

Figura 5. a. La casa real inaugura la nueva sala del Museo en el que se encuentra el montaje del esqueleto de Diplodocus carnegii, regalo realizado a su Majestad Alfonso XIII. b. Detalle de la misma donde se observa, en primer plano y de izquierda a derecha, a Ángel Cabrera, Francisco Ferrer, Ignacio Bolívar (director del MNCN), la Reina María Cristina y la Infanta Beatriz. Al fondo, a la izquierda, se distingue a Eduardo Hernández-Pacheco, jefe de la Sección de Mineralogía y Geología del MNCN. Archivo General de Palacio, Palacio Real. No Inv.: 10164235. Esta imagen fue publicada en $A B C$ el 3 de diciembre de 1913 y en la revista Blanco y Negro el 7 diciembre de 1913 (Anónimo, 1913b, 1913f). Copyright (C) Patrimonio Nacional. a. Royal Household opening the new hall of the MNCN with the skeleton of Diplodocus carnegii, a gift made to King Alfonso XIII. $\boldsymbol{b}$. Detail of the same photograph. In the foreground, from left to right, Ángel Cabrera, Francisco Ferrer, Ignacio Bolivar (MNCN director), the Queen María Cristina and the Infanta Beatriz. At the back of the room, on the left, Eduardo Hernández-Pacheco, head of the Section of Mineralogy and Geology of the MNCN. Archivo General de Palacio, Palacio Real, inventory number: 10164235. This image was published in $\mathrm{ABC}$ on December $3^{\text {th }}$, 1913, and in $\mathrm{Blanco} \mathrm{y}$ Negro on December $7^{\text {th }}, 1913$ (Anónimo, 1913b, 1913f). Copyright C Patrimonio Nacional. 


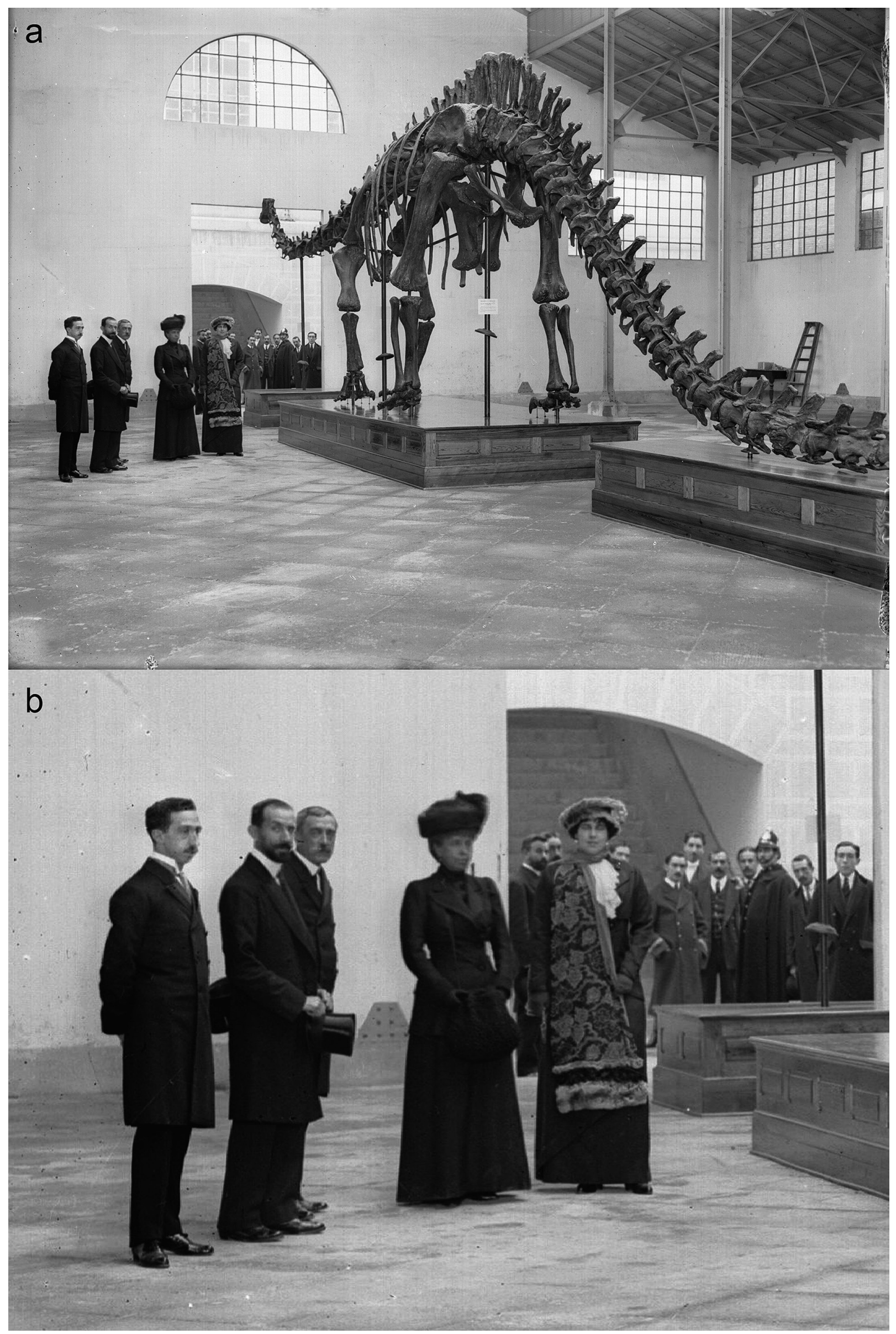


Dentro de los actos oficiales, el Rey Alfonso XIII concede distinciones honoríficas a Carnegie, Holland y Coggeshall, "queriendo significar el alto aprecio que le ha merecido el generoso proceder del donante y los trabajos realizados por los Ayudantes del citado Museo" (ACN0275/017, doc.15, doc.16). Por parte del MNCN se envía una carta de agradecimiento a Holland por los servicios prestados, pidiendo que acepte un obsequio como expresión de agradecimiento: "sirva de marco, esa reproducción de una portada de la Alhambra al retrato de su Señora que siempre le acompaña" (ACN0303/002/001, doc.60). Holland agradece el regalo y el trato recibido durante su estancia (ACN0303/002/001, doc.61). Bolívar agradece a Carnegie, en nombre del personal del MNCN, la donación del "colosal esqueleto" del "gran saurio jurásico" (ACN0303/002/001, doc.63). Por su parte y antes de su par- tida, Holland y Coggeshall posan en una fotografía con algunos de los miembros más destacados del MNCN (Fig. 6).

Sin embargo, a principios de diciembre y ante las " $m a$ nifestaciones de desagrado contra los que pasan a visitar el pabellón donde está instalado el Diplodocus" por parte de los alumnos de la Escuela de Ingenieros Industriales, Bolívar envía una carta al Director de La Escuela de Industriales en la que, dando constancia de este hecho, acepta que el público visite únicamente el pabellón donde se ha instalado el Diplodocus en el horario sugerido por el director de esta Escuela, estando cerrado el resto del tiempo con el pretexto de que se realizan obras para instalar las colecciones de Paleontología y Geología en dicha sala (ACN0303/002/001, doc.62).

Mientras tanto, es Ángel Cabrera quien se encarga de desmentir ante la prensa algunos de los falsos rumores que

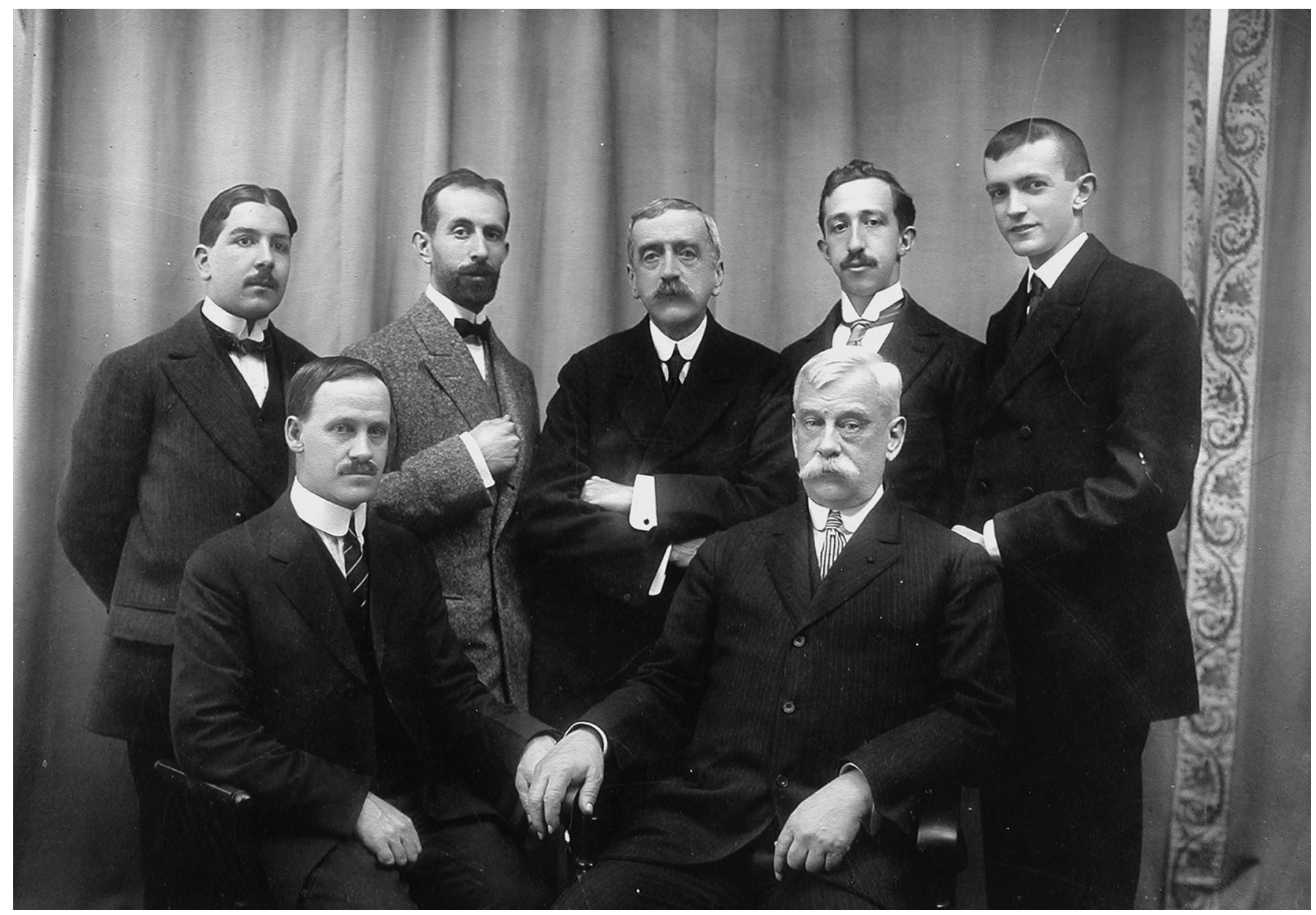

Figura 6. Finalizada la instalación del esqueleto de Diplodocus, William J. Holland (sentado a la derecha de la fotografía) y Arthur Coggeshall (sentado a la izquierda), posan para una fotografía junto con algunos miembros del Museo. De pie, de izquierda a derecha se sitúan Luis Lozano, Francisco Ferrer, Ignacio Bolívar Urrutia, Ángel Cabrera y Cándido Bolívar Pieltaín (Foto: ACN F7C1).

When the installation of the skeleton of Diplodocus was finished, William J. Holland (seated at the right of the photograph) and Arthur Coggeshall (seated at the left), posed for a photograph with the staff of the MNCN. Standing, from the left to the right are Luis Lozano, Francisco Ferrer, Ignacio Bolivar Urrutia, Angel Cabrera and Candido Bolivar Pieltaín (Photo: ACN F7C1). 
existen en relación con el Diplodocus, como que se trate de un feroz carnicero, que el ejemplar original se conserve en el Museo de Nueva York o que la reproducción regalada a Alfonso XIII sea la tercera realizada (Cabrera 1913b) (Fig. 7). A pesar de haber dedicado toda su vida al estudio de los mamíferos, será curiosamente Cabrera quien descubra y describa, en 1947, el primer dinosaurio jurásico conocido en América del Sur, el saurópodo Amygdalodon patagonicus (ver Magnussen Saffer, 2007).

En la Sesión del 4 de Febrero de 1914 de la Real Sociedad Española de Historia Natural se solicita a Hernández Pacheco que prepare una conferencia sobre el hallazgo, montaje y "particularidades (sic) dignas de ser conocidas" de Diplodocus carnegii (Pérez Zúñiga, 1914). Pacheco acepta, proponiendo también la redacción de una nota sobre este asunto (Hernández-Pacheco, 1914). No queda documentación alguna en la que quede constancia de que estas propuestas fueran cumplidas.

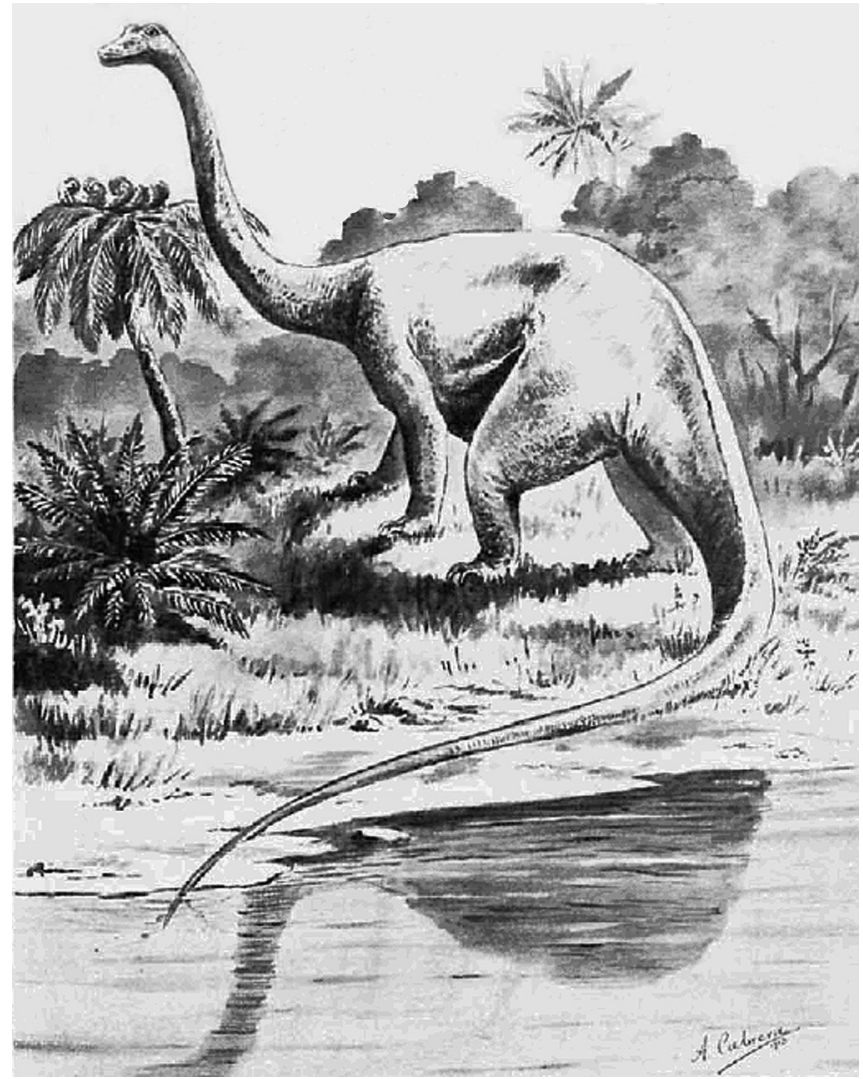

Figura 7. Interpretación del aspecto que tendría el Diplodocus en vida, realizada por Ángel Cabrera y publicada en La Ilustración Española y Americana el 8 de diciembre de 1913 (Cabrera, 1913a). Por cortesía de la Hemeroteca Nacional (BNE).

Interpretation of Diplodocus would look at life, by Ángel Cabrera. This picture was published in La Ilustración Española y Americana, December $8^{\text {th }}$, 1913 (Cabrera, 1913a). Courtesy of the Hemeroteca Nacional of Spain (BNE).
Dos meses más tarde, el 14 de febrero de 1914, Alfonso XIII envía desde Sevilla un retrato con su autógrafo a Carnegie (Fig. 8). Además lo acompaña con una carta en la que el monarca agradece esta gran contribución a las colecciones científicas de España y a la cultura de este país. Curiosamente, afirma que fue él quien dio las órdenes para que fuera instalado en el MNCN, donde contribuirá a la difusión del conocimiento mejor de lo que podría haberlo hecho en Palacio (Anónimo, 1914a, 1914b).

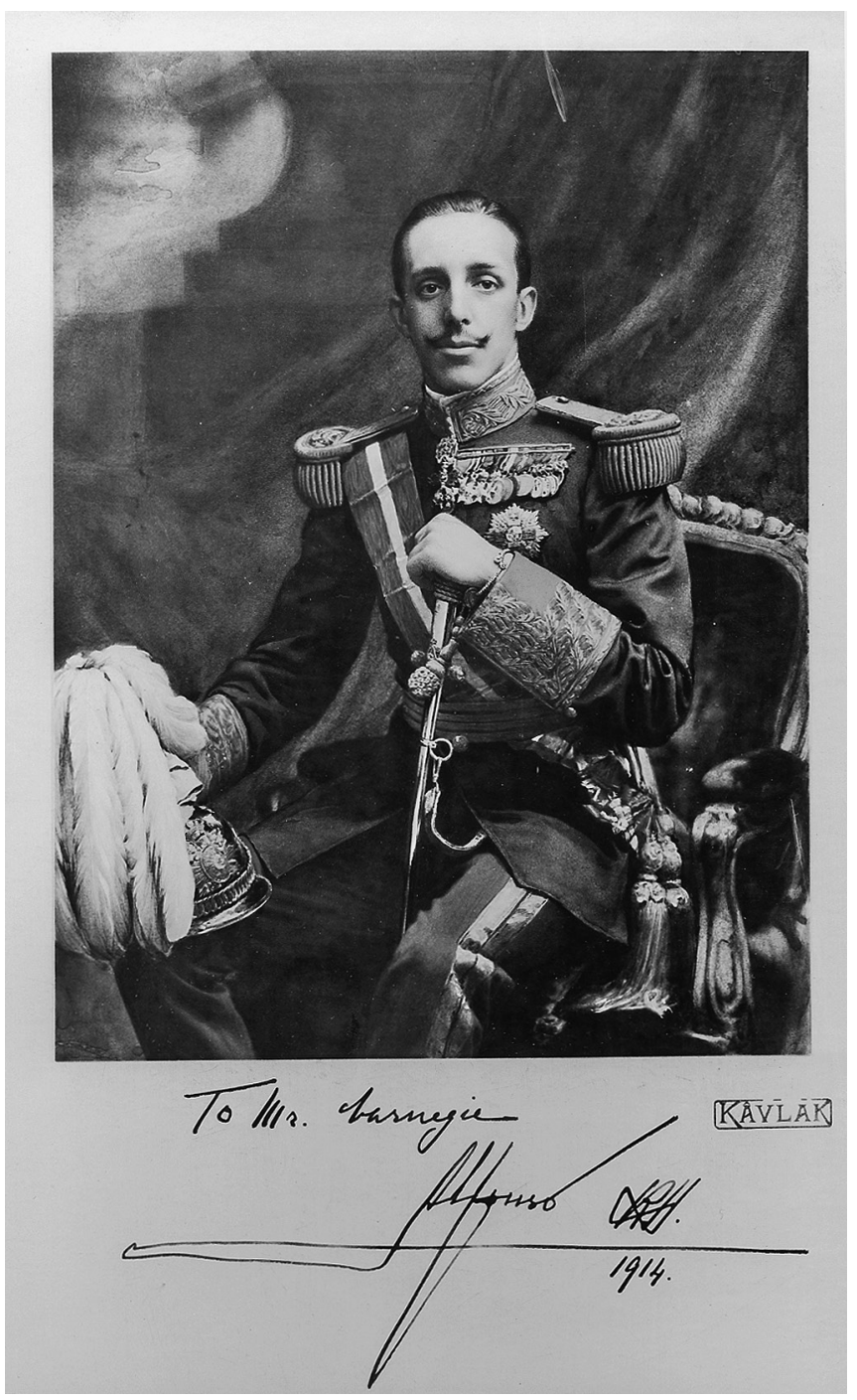

Figura 8. Retrato de Alfonso XIII enviado a Andrew Carnegie, en febrero de 1914, como agradecimiento por el regalo del esqueleto de Diplodocus. La fotografía está firmada y dedicada por el monarca. Cortesía de la Carnegie Library of Pittsburgh. Pennsylvania Dept. Andrew Carnegie benefactions collection, CA-537. Portrait of Alfonso XIII sent to Andrew Carnegie, in February 1914, in gratitude for the gift of the skeleton of Diplodocus. The photograph is signed and dedicated by the monarch. Courtesy of Carnegie Library of Pittsburgh. Pennsylvania Dept. Andrew Carnegie benefactions collection, CA-537. 


\section{EL TRASLADO DEL ESQUELETO DE DIPLODOCUS A LA ACTUAL SALA DE PALEONTOLOGÍA}

Unos meses más tarde se publica en el Boletín de Instrucción Pública la aprobación, por parte del Rey, del proyecto para que el arquitecto Enrique Repullés y Segarra establezca la comunicación entre el Pabellón en el que ha quedado instalado el esqueleto de Diplodocus y el edificio que ocupaba el resto del MNCN, consiguiendo así la independencia entre esta Institución y la Escuela de Ingenieros Industriales, propuesta por la que tanto había luchado Bolívar. El proyecto se aprobó por un importe total de 4.558,46 pesetas (Boletín de Instrucción Pública de $\mathrm{N}^{\circ}$ 51, publicado el 26 de junio de 1914). Un mes después se aprueba otro presupuesto de $9.883,78$ pesetas para que este mismo arquitecto realice las reparaciones pertinentes en el patio donde está instalado el esqueleto de Diplodocus y para que se adecuen las dependencias anejas al mismo como Laboratorio de Prácticas (Boletín de Instrucción Pública de $\mathrm{N}^{\circ}$ 60, publicado el 28 de julio de 1914). Un año más tarde continúan los arreglos en dicho Salón, donde están depositadas las colecciones de Paleontología y Geología, con el objetivo de poder mostrar al público el mayor número de ejemplares posibles (Bolívar y Urrutia, 1915). No obstante, sólo una pequeña parte de las colecciones pudo ser finalmente expuesta al público debido a que éstas se encontraban depositadas en armarios cerrados en el mismo pabellón alrededor del Diplodocus, cumpliendo la sala tanto las funciones de sala de exposición como de almacén (Montero, 2003).

El esqueleto de Diplodocus de Madrid no sólo pasó a formar parte del ámbito científico, nombrándose en publicaciones españolas como ejemplo de uno de los saurópodos mejor conocidos (Royo y Gómez, 1926), sino que su fama se hizo extensiva a toda la población, siendo usado por la prensa como símbolo de estabilidad, grandeza y gran valor económico, aunque también fuera empleado como elemento de burla política (Anónimo, 1913d, 1918; Nardy, 1933), tal como se había afirmado ya antes de su instalación: "La monstruosa armadura servirá para estudio de los hombres de ciencia; para que los autores del género chico le coloquen al respetable público unas cuantas coplas intencionadas y alusivas a la política, y para que тиchos curiosos espectadores, a imitación de aquel rey que cuando vió por primera vez el mar no se le ocurrió decir más que “¡Cuánta agua!”, exclamen: “¿Pero qué grandes animales eran los antediluvianos!”” (Anónimo, 1913a).

Por su parte, Andrew Carnegie fue un personaje bien conocido en España dada la envergadura y la repercusión social y política de su regalo, por lo que la noticia de su fallecimiento, el 11 de agosto de 1919, no pasó desapercibida, apareciendo publicada en la prensa española, en la que de nuevo se recordaba el extraordinario obsequio que había hecho al Rey Alfonso XIII (Anónimo, 1919a, 1919b).
En los primeros meses de 1928, Miguel Primo de Rivera, presidente del Gobierno militar, en ese momento Directorio Civil, realizó una visita al MNCN. Tras comprobar la urgente necesidad de más espacio para exponer y almacenar las colecciones, prometió realizar los trámites necesarios para desalojar del edificio, tanto la parte ocupada por la Escuela de Ingenieros como la correspondiente al cuartel de la Guardia Civil, alojándolos en un nuevo inmueble junto a la Residencia de Estudiantes, lo que permitiría realizar la ampliación del Museo (Peña, 1928). Este proyecto fue difundido por la prensa (Anónimo, 1928). A finales de año se consigue la ampliación aunque ésta ocurre de otra manera: el 10 de diciembre de 1928 se comunica por Real Orden que parte de los locales que ocupaba el Museo del Traje Regional en el Palacio de Industrias del Paseo de la Castellana, es decir, en el mismo edificio que el MNCN, que habían sido desalojados ese mismo año por su traslado al antiguo Hospicio, se entrega al MNCN para su ampliación (ACN, Administración, 1928). Otra parte de éstos locales es ocupada por la Guardia Civil (Anónimo, 1935a).

El traslado de ejemplares a los nuevos locales, que afectaba también al esqueleto de Diplodocus, no se realiza hasta 1935, cerrándose el Museo al público el año anterior para la ejecución de las obras de mejora y ampliación (Anónimo, 1934) (Fig. 9). También en los periódicos se habla de este hecho, en los que abundan las noticias con este animal como protagonista. Por ejemplo, el 5 de marzo de 1935, en el periódico El Sol se informa de que la Sala de Diplodocus está cerrada al público para preparar el traslado de este animal "terrible y monstruoso", "la maravilla de las maravillas para grandes y pequeños", cuyo tamaño se afirma que es mayor que el de King-Kong, " $a$ una más espaciosa sala donde exhibirse a la admiración de las gentes", que se encuentra en ese momento en obras (Prats, 1935). En septiembre, las obras y traslado de las colecciones de Geología, Prehistoria y Paleontología están muy avanzadas, pretendiendo su inauguración durante la primera semana de octubre (Llorens, 1935; Anónimo, 1935b). Lamentablemente, en octubre, con las nuevas salas casi terminadas, por falta de financiación para adquirir los muebles necesarios para la exposición de los ejemplares y finalización de las obras dirigidas por el Arquitecto Manuel Sánchez Arcas, se despide a todos los que trabajan en ellas, retrasándose la inauguración (ACN, Administración, 1935). Estas obras dividen el único salón existente hasta el momento, de techo muy alto, en la distribución que permanece en la actualidad, con tres pisos, en la que el Diplodocus se sitúa en el piso central. Aunque su traslado supuso un trabajo laborioso y delicado, se realizó sin grandes incidentes gracias a que había personal del MNCN que ya había estado presente en el montaje de 1913, como José Royo y Gómez, que en ese momento ocupa la dirección de la sección de Paleontología del Museo. Poco tiempo después se abrió al público la nueva ampliación de Geología y Paleontología del Museo. De esta manera, 


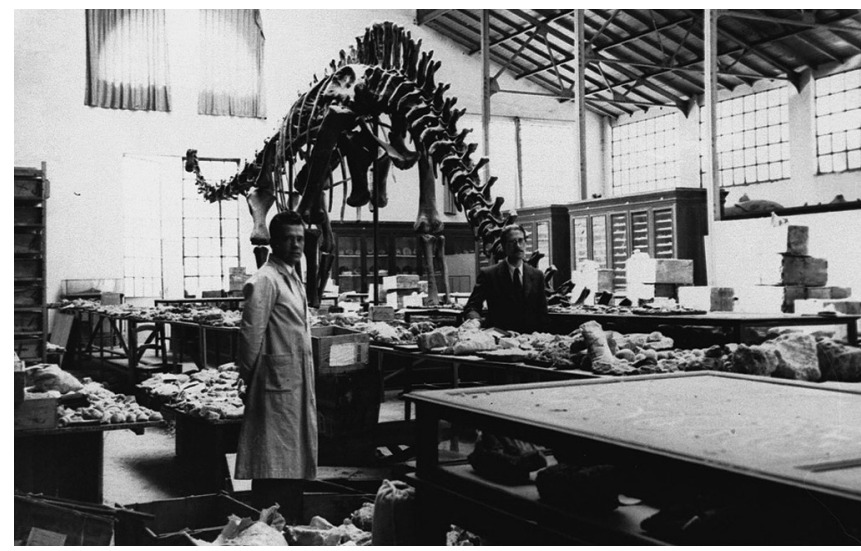

Figura 9. Aspecto de la sala de Paleontología con el esqueleto de Diplodocus montado tal como permaneció hasta 1935. Al fondo se distinguen los armarios que contienen el resto de las colecciones. Fotografía tomada por Royo y Gómez en la que se distingue a Vicente Sos Baynat en agosto de 1934, cuando se produce el desmantelamiento de la sala para su traslado a los nuevo locales (Foto: ACN 3159).

Aspect of the Paleontology room with the skeleton of Diplodocus, as it remained until 1935. In the background are the cabinets containing the rest of the collections. Picture taken by Royo y Gómez, where it is seen Vicente Sos Baynat in August 1934, when the collections are being prepared for transfer to the new rooms (Photo: ACN 3159).

el esqueleto de Diplodocus quedó definitivamente ubicado en el lugar donde continúa en la actualidad.

Casi cincuenta años más tarde, en 1984, se solicita al Carnegie Museum of Natural History el envío de las réplicas de algunas piezas que se habían perdido o dañado con los años. Los elementos que se habían perdido eran algunos chevrones, las últimas ocho vértebras caudales y algunas falanges de los cuatro autópodos. Además, los elementos que tenían que ser sustituidos por estar deteriorados eran algunas vértebras, casi todas ellas dorsales. Sin embargo, debido al paso del tiempo los moldes del Carnegie Museum of Natural History habían quedado inutilizables, por lo que estas piezas se encargaron finalmente al British Museum of Natural History. El único cambio importante realizado respecto al montaje original de Diplodocus fue la sustitución, durante la última gran obra de remodelación de la Sala en 19881989, de la peana de madera, que tanto había costado diseñar, encargar y construir, por un enrejado metálico moderno.

\section{UN SEGUNDO REGALO DEL CARNEGIE MUSEUM}

En el Acta de la Sesión de la Junta de Profesores de los Museos de Historia Natural del día 9 de noviembre de
1935, José Royo y Gómez informa sobre el envío de una réplica del cráneo y de la mandíbula de un nuevo ejemplar de Diplodocus por parte del Carnegie Museum of Natural History de Pittsburg. Este nuevo ejemplar, que había sido hallado por dicha institución, era considerado " $m u$ cho más completo que el que tiene el esqueleto existente en el Museo" (ACN0311/002). El ejemplar original, CN 11161, había sido encontrado en el Dinosaur National Monument (Utah) por Douglass en 1912 y descrito y figurado por Holland en 1924 (Holland, 1924). Posteriormente este cráneo se atribuyó a Diplodocus longus Marsh, 1878 (Mc Intosh, 1981). Aunque en un principio se plantea la posibilidad de sustituir este cráneo por el que posee el esqueleto montado, finalmente se decide exponerlo al público junto a éste, cosa que se realiza ese mismo año (Anónimo, 1935b). El 16 de noviembre de 1935 se agradece la donación, en nombre de la Junta Directiva del MNCN, a Andrew Avinoff, en ese momento director del Carnegie Museum (ACN0275/017, doc.18; ACN0275/018). Esta nueva réplica estuvo expuesta junto con el esqueleto original hasta la última remodelación de la sala en 1989. Sigue formando parte de la colección actual del Museo Nacional de Ciencias Naturales con el número de catálogo MNCN 66288 (Fig. 10). Por su parte, el esqueleto siguió siendo un objeto sugerente para la sociedad, realizándose caricaturas y hasta cuentos para niños. También el mundo del arte cinematográfico hizo un guiño a este esqueleto, siendo el protagonista de la canción "El Diplodocus", incluida en el exitoso largometraje "Acompáñame", producido en 1966 y protagonizado por Rocío Durcal.

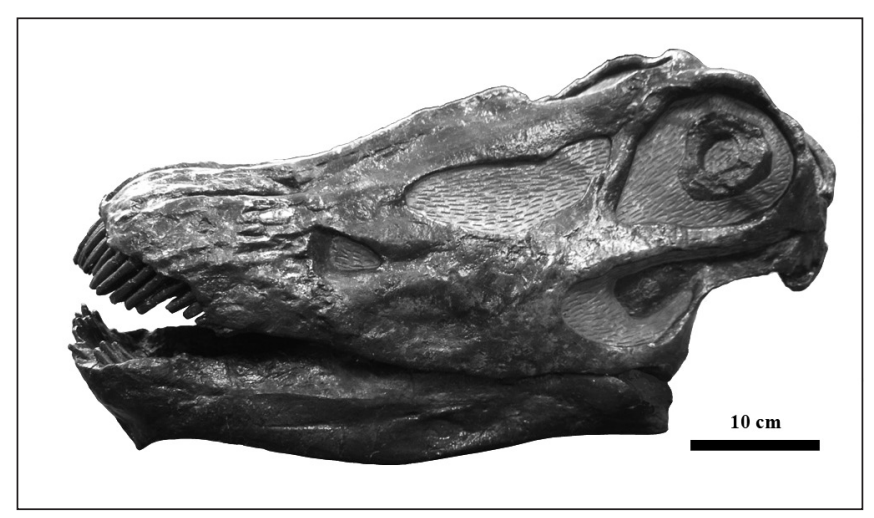

Figura 10. Réplica del segundo cráneo de Diplodocus que fue regalado al MNCN por el Carnegie Museum of $\mathrm{Na}$ tural History de Pittsburgh en 1935, tal como se conserva en la actualidad. MNCN 66288.

Replica of the second skull of Diplodocus, which was donated to the MNCN by the Carnegie Museum of Natural History of Pittsburgh in 1935, as preserved in the present. Catalogue number MNCN 66288. 


\section{CONCLUSIONES}

Aunque se conocían las circunstancias que rodearon la cesión de las réplicas de Diplodocus por el magnate americano Andrew Carnegie y su repercusión en varios países, tales como Inglaterra o Argentina, en España apenas se disponía de información sobre este hecho. En este trabajo se da a conocer por primera vez una gran cantidad de información inédita que explica las razones por las cuales el Museo Nacional de Ciencias Naturales dispone de una de las nueve copias del esqueleto de Diplodocus realizadas en las dos primeras décadas del siglo XX. Se constata que la inauguración de la Sala del Diplodoco fue realizada por parte de la Casa Real en diciembre de 1913, siendo este uno de los acontecimientos que más repercusión social tuvo en un momento en el que el país hacía fuertes críticas a su clase política.

La expectación que se generó, así como la problemática y trascendencia de este regalo, se ponen de manifiesto en los continuos artículos de toda índole publicados en la prensa española entre 1913 y 1935, año en el que tiene lugar su traslado a la nueva sala.

El conocimiento de los pormenores de la llegada e instalación de Diplodocus a Madrid ha servido, además, para fechar documentación manuscrita e iconográfica, depositada tanto en el MNCN como en otras instituciones públicas, ya que en muchos casos se encontraba sin identificación.

El Diplodocus del MNCN, único esqueleto de un dinosaurio instalado España durante siete décadas, permitió acercar la Paleontología a la población de este país, siendo un elemento no solo apreciado por la comunidad científica sino también conocido y valorado en todos los sectores sociales.

\section{AGRADECIMIENTOS}

Los autores agradecen la revisión del artículo a los doctores José Luis Sanz (Universidad Autónoma de Madrid) y a Ángel Montero (Jardín Botánico de Córdoba). Este trabajo ha sido realizado mediante un contrato I3P (A.P.G.) en la colección de Paleontología de Vertebrados del MNCN durante 2008 y mediante el disfrute de una beca del subprograma FPU del Ministerio de Ciencia e Innovación (ref. AP2007-00873) en 2009. El acceso a la documentación necesaria se ha conseguido gracias a la ayuda recibida por parte del personal del Archivo del MNCN (Carmen Velasco, Manuel Parejo y Noelia Cejuela). Nuestra más sincera gratitud por el interés mostrado y el tiempo dedicado. Asimismo, agradecemos al personal del Archivo del Palacio Real de Madrid, muy especialmente a Antonio Alonso Zimmerli, así como al personal de la Biblioteca Nacional (Hemeroteca) las facilidades prestadas para el acceso a la documentación utilizada en este artículo. Gil Pietrzak, de la Carnegie Library of Pittsburg, facilitó el retrato del rey Alfonso XIII con la dedicatoria a Mr. Carnegie.

\section{REFERENCIAS}

Anónimo. 1899. Otro monstruo antediluviano. El animal más fuerte que ha habido en el mundo. Alrededor del Mundo, 29 de septiembre, 8-9.

Anónimo. 1900a. Una lucha de monstruos antediluvianos. Alrededor del Mundo, 15 de febrero, 5.

Anónimo. 1900b. El Mundo Naval Ilustrado, 20 de diciembre, 34, 540 .

Anónimo. 1913a. El "Dioprodocus". ABC, 12 de noviembre, 6.

Anónimo. 1913b. En el Museo de Historia Natural. ABC, 3 de diciembre, 3 .

Anónimo. 1913c. Conferencia del Dr. Holland. El imparcial, 29 de noviembre, 5.

Anónimo. 1913d. El "Monteroprodocus". El duende, 23 de noviembre, 9 .

Anónimo. 1913e. La Nochebuena de Don Homobono. El imparcial, 28 diciembre, 1.

Anónimo. 1913f. Visita a un Museo. Blanco y Negro, 7 de diciembre, 29.

Anónimo. 1913g. Un regalo de Carnegie. El imparcial, 12 de noviembre, 5 .

Anónimo. 1914a. El viaje de los reyes. El imparcial, 15 de febrero, 4.

Anónimo. 1914b. Fin de jornada. Regreso de los reyes. $A B C$, 15 de febrero, 7.

Anónimo. 1918. Las mentiras de "Alrededor del Mundo". Alrededor del Mundo, 29 de julio, 8-9.

Anónimo. 1919a. Muerte del gran filántropo Andrés Carnegie. El imparcial, 13 de agosto, 1.

Anónimo. 1919b. Figuras mundiales. Andrés Carnegie. La Ilustración Española y Americana, 15 de agosto, 9.

Anónimo. 1928. Va a ser ampliado el Museo de Ciencias. El Debate, 18 de octubre, 5.

Anónimo. 1934. El Museo de Historia Natural será ampliado próximamente. Ahora, 14 de septiembre, 27.

Anónimo. 1935a. Reformas en el Museo de Ciencias Naturales. $Y A, 30$ de abril, 7 .

Anónimo. 1935b. Pasado mañana se abre el Museo de Ciencias Naturales. El Debate, 29 de septiembre, 17-18.

Bolívar y Urrutia, I. 1915. Discurso de Recepción del Ilmo. Sr. D. Ignacio Bolívar y Urrutia en la Real Academia de Ciencias Exactas, Físicas y Naturales. La Escuela Moderna, 1 de Noviembre, 725-733.

Cabrera, A. 1913a. La historia del "Diplodocus". La Ilustración Española y Americana, 45, 346-347.

Cabrera, A. 1913b. El Diplódoco y su esqueleto. Alrededor del Mundo, 14 de diciembre, 7-8.

Díaz del Villar, J.M. 1913. Sesión extraordinaria del 28 de Noviembre. Boletín de la Real Sociedad Española de Historia Natural, 13, 31.

Egozcue, J. 1873. Noticia sobre la existencia en España de restos fósiles de Megalosaurus y de Hyaena spelaea y brunnea. Anales de la Sociedad Española de Historia Natural, Actas, 2, 29-31.

Espinar Moreno, M. 2002. Notas sobre la vida y obras del R. P. Manuel M. Sánchez-Navarro Neumann, S. J. (1867- 
1941). In: Historia del Observatorio de Cartuja, 19022002. Nuevas Investigaciones (coord. M. Espinar, J. A. Esquivel \& J. A. Peña). Instituto Andaluz de Geofísica. Excelentísimo Ayuntamiento de Granada, 40-68.

Ferrando, P. 1912. Sesión del 28 de Febrero, Sección de Zaragoza. Boletín de la Real Sociedad Española de Historia Natural, 12, 188.

Hatcher, J.B. 1901. Diplodocus (Marsh), its osteology, taxonomy, and probable habits, with a restoration of the skeleton. Memories of the Carnegie Museum, 1, 1-63.

Hernández Pacheco, E. 1914. Sesión del 4 de febrero. Boletín de la Real Sociedad Española de Historia Natural, 14, 91.

Holland, W.J. 1900. The vertebral formula in Diplodocus. Science, 11, 816-818.

Holland, W.J. 1924. The Skull of Diplodocus. Memories of the Carnegie Museum, 9, 379-403.

Llorens, M.G. 1935. Charlas faunícolas.- El Arca Varada. Ahora, 7 de septiembre, 35 .

Lorenzo, F. 1913. El perfil del día. El Imparcial, 13 de noviembre, 3 .

Magnussen Saffer, M. 2007. Ángel Cabrera. Un brillante Mastozoólogo y su contribución a los Mamíferos Fósiles. Paleo, Boletín Paleontológico, 28, 18-21.

Marsh, O.C. 1878. Principal characters of American Jurassic dinosaurs. Part 1. American Journal of Science, 16, 411-416.

Mc Intosh, J.S. 1981. Annotated Catalogue of the Dinosaurs (Reptilia, Archosauria) in the collections of Carnegie Museum of Natural History. Bulletin of Carnegie Museum of Natural History, 18, 68 pp.

Montero, A. 2003. La paleontología y sus colecciones desde el Real Gabinete de Historia natural al Museo Nacional de Ciencias Naturales. Monografías (Museo Nacional de Ciencias Naturales), Madrid, 383 pp.

Nardy, G. 1933. Fémina. El ocaso de las joyas. Nuevo Mundo, 13 de octubre, 6-7.
Peña, H. de la. 1928. Las riquezas que guarda el Museo están expuestas a perderse. Nuevo Mundo, 1 de junio, 11-13.

Prats, A. 1935. En la instalación del Museo de Ciencias Naturales se están realizando importantes reformas. El Sol, 5 de marzo, 2.

Pérez García, A., Sánchez Chillón, B. \& Ortega, F. 2009. Aportaciones de José Royo y Gómez al conocimiento sobre los dinosaurios de España. Paleolusitana, 1, 339-364.

Pérez Zúñiga, E. 1914. Sesión del 4 de Febrero. Boletín de la Real Sociedad Española de Historia Natural, 14, 91.

Rivero. 1913. El director del Instituto Carnegie dirigiendo la instalación del "Diplodocus" en el Museo de Ciencias Naturales. Una reproducción del "Diplodocus" en Madrid. La Ilustración Española y Americana, 45, 347.

Royo y Gómez, J. 1925. Resultados científicos obtenidos en un viaje por Francia, Suiza, Alemania y Bélgica. Boletín de la Real Sociedad Española de Historia Natural, 25, 114-117.

Royo y Gómez, J. 1926. Los descubrimientos de reptiles gigantescos en Levante. Boletín de la Sociedad Castellonense de Cultura, 7, 147-162.

Sánchez Chillón, B. \& Pérez García, A. 2008. Los Ictiosaurios del Museo Nacional de Ciencias Naturales. Los primeros reptiles que llegaron a la Colección de Paleontología. Periódico del MNCN, 8, 10.

Sánchez-Navarro Neumann, M.M. 1911. La segunda Asamblea general de la Asociación Internacional de Sismología. Boletín de la Real Sociedad Española de Historia Natural, 11, 470-473.

Vilanova y Piera, J. 1872. Compendio de Geología. Imprenta de Alejandro Gómez Fuentenebro, Madrid, 588 pp.

Vilanova y Piera, J. 1873. Sesión del 5 de febrero. Anales de la Sociedad Española de Historia Natural, Actas, 2, 8.

Manuscrito recibido: 5 de Junio, 2009 Manuscrito aceptado: 30 de Octubre, 2009 


\section{Anexo 1: Documentación inédita del ACN}

ACN0135/007/006. 1925. Dibujo de uno de los esqueletos de Iguanodon del Museo de Historia Natural de Bruselas, realizado por Royo y Gómez.

ACN0275/017. 1913-1935. Expediente por el que Carnegie obsequia al Rey con el envío al Museo de Ciencias Naturales de un esqueleto de Diplodocus, preparado por el director del Museo Carnegie, y las circunstancias sobre su traslado y su instalación en el Museo, 18 doc., 36 pp.

ACN0275/018. 1935. La Junta Directiva del Museo de Ciencias Naturales agradece la donación de un cráneo de Diplodocus, expuesto en la sala de Paleontología, junto al esqueleto regalado por Carnegie, 1 doc., 1 p.

ACN0303/001. 1911. El Museo de Ciencias Naturales de Madrid solicita el molde del iguanodón y el de diplodocus de Pittsburg, 5 doc., 9 pp.

ACN0303/002. 1911-1913. Expediente por el que Carnegie obsequia al Rey con el envío al Museo de Ciencias Naturales de un esqueleto de diplodocus, preparado por el director de dicho museo y las circunstancias sobre su traslado e instalación en el museo, 67 doc., 111 pp.
ACN0303/002/022. 1913. En el vapor Montserrat de la Compañía Transatlántica ha llegado a Barcelona el ejemplar del Diplodocus. La Época, 9 de octubre, p. 55

ACN0303/002/054. 1913. El Diplodocus. El Mercantil Valenciano, 15 de noviembre, p. 94.

ACN0311/002. 1931-1939. Libro de Actas de las Sesiones celebradas por la Junta de Profesores de los Museos de Historia Natural (Museo Nacional de Ciencias Naturales, Jardín Botánico y Museo Antropológico), 1 doc., 111 pp. ACN. Administración, 1928. R.O. nº 69 de 10 de diciembre, por la que se dispone que los locales que ocupaba el Museo del Traje Regional el en Palacio de Industrias del Paseo de la Castellana sean entregadas a la Dirección del Museo de Ciencias Naturales para la ampliación y complemento se sus instalaciones, $1 \mathrm{p}$.

ACN. Administración, 1935. Carta enviada al Sr. Rector con fecha de 10 de Octubre por la que se comunica el retraso en la apertura de la Salas de Geología, Prehistoria y Paleontología por falta de presupuesto, $1 \mathrm{p}$. 